\title{
Prevalence and predictors associated with in-hospital mortality in acute ST segment elevation myocardial infarction after reperfusion therapy in developing country
}

\author{
Chorchana Wichian $^{1 \#} \wedge$, Thotsaporn $_{\text {Morasert }^{2 \#} \wedge}$, Thanapon Nilmoje $^{3 \wedge}$, Ply Chichareon $^{3 \wedge}$ \\ ${ }^{1}$ Cardiology Unit, Department of Internal medicine, Suratthani Hospital, Suratthani, Thailand; ${ }^{2}$ Pulmonary and Critical Care Medicine Unit, \\ Department of Internal Medicine, Suratthani Hospital, Surat Thani, Thailand; ${ }^{3}$ Cardiology Unit, Department of Internal Medicine, Faculty of \\ Medicine, Prince of Songkla University, Songkhla, Thailand \\ Contributions: (I) Conception and design: C Wichian, T Morasert, P Chichareon; (II) Administrative support: C Wichian, T Morasert, T Nilmoje; \\ (III) Provision of study materials or patients: C Wichian; (IV) Collection and assembly of data: C Wichian; (V) Data analysis and interpretation: All \\ authors; (VI) Manuscript writing: All authors; (VII) Final approval of manuscript: All authors. \\ "These authors contributed equally to this work. \\ Correspondence to: Chorchana Wichian, MD. Interventional Cardiologist, Department of internal medicine, Suratthani Hospital, Surrathani 84000, \\ Thailand. Email: chorchana@gmail.com.
}

Background: The prevalence and predictors of in-hospital mortality in the unselected patients with STsegment elevation myocardial infarction (STEMI) after reperfusion therapy with fibrinolytic or primary percutaneous coronary intervention (PCI) in developing country have not well established.

Methods: We conducted a retrospective cohort study design. All admission records of acute ST elevation myocardial infarction at Suratthani hospital between October 2015 and September 2017.

Results: The finding between October 2015 and September 2017, a total of 431 patients received reperfusion therapy and were included in the present study. Majority of the patient were male $(80.5 \%)$, almost half of the population had anterior wall MI, 32 patients died (7.4\%), and 399 survived during the index hospitalization. The prevalence of chronic kidney disease, anterior wall MI, cardiogenic shock and cardiac arrest in non-survived group was higher than the survived group. Echocardiogram was performed in 299 patient (69.4\%) and the median left ventricular ejection fraction (LVEF) in overall population was $51 \%$. After adjustment for baseline variables, LVEF (aOR 0.91, 95\% CI, 0.83-0.98, P=0.02) and cardiac arrest at presentation (aOR 22, 95\% CI, 1.22-410.05, $\mathrm{P}=0.036$ ) were independently associated with in-hospital mortality.

Conclusions: LVEF and cardiac arrest at presentation were independently associated with in-hospital mortality in STEMI patients receiving reperfusion therapy either fibrinolytic therapy or primary PCI.

Keywords: Predictor of in-hospital mortality; acute myocardial infarction (MI); reperfusion therapy; echocardiogram

Submitted Mar 29, 2020. Accepted for publication Aug 30, 2020.

doi: $10.21037 / \mathrm{cdt}-20-398$

View this article at: http://dx.doi.org/10.21037/cdt-20-398

^ ORCID: Chorchana Wichian, 0000-0001-8929-644X; Thotsaporn Morasert, 0000-0002-3353-2033; Thanapon Nilmoje, 0000-0001-7061-5789; Ply Chichareon, 0000-0002-3650-8661. 


\section{Introduction}

Acute myocardial infarction (MI) is a clinical spectrum caused by an obstruction of coronary blood flow resulting in a damage of myocardium. The classification can be subdivided to ST segment elevation (STEMI) or nonST-segment elevation myocardial infarction (NSTEMI) according to a presenting electrocardiogram (1).

The incidence of STEMI is still high, 58 per 100,000 per year in European STEMI registry in Sweden (2). Proportion of STEMI in patients included in the Thai acute coronary syndrome registry was 55 percent and carried 5 percent inhospital to 14 percent 1-year mortality rate, respectively (3).

The reperfusion therapy with either fibrinolytic agents or primary percutaneous coronary intervention (PCI) are performed to restore myocardial blood flow and to optimize myocardial salvage. Although reperfusion treatment improves survival of STEMI patients but the mortality after reperfusion is still high in the current practice.

The clinical predictors before and during treatment may help to identify high-risk STEMI patient who would benefit from an aggressive management. Elderly, previous myocardial infarction (MI), history of heart failure, increased pulse rate and lower systolic blood pressure at presentation, elevated of initial serum creatinine and cardiac biomarker levels, ST segment depression on presenting electrocardiogram, and not having a PCI, were the independent predictors for 6-month mortality in The Global Registry of Acute Coronary Events (GRACE) (4) and the previous report of Thrombolysis in Myocardial Infarction Risk score (TIMI) permit a fairly precise determination of the short-term mortality (5)

Echocardiography is recommended routinely to measure left ventricular ejection fraction (LVEF) in STEMI patient during hospitalization (6). LVEF may be improved with reperfusion therapy (7). There were limited number of studies to determine prognostic value of LVEF for inhospital mortality in STEMI patients.

The objective of the study was to identify prevalence and factors associated with in-hospital mortality in acute STEMI patients after reperfusion. We present the following article in accordance with the TRIPOD reporting checklist (available at http://dx.doi.org/10.21037/cdt-20-398).

\section{Methods}

\section{Study design and setting}

The study was a retrospective cohort design conducted in
Surat Thani Hospital, a tertiary care center in the South of Thailand. The study was conducted in accordance with the Declaration of Helsinki (as revised in 2013). The ethical committee of Surat Thani Hospital, Thailand, approved the study protocol (No.19/2020) and individual consent for this retrospective analysis was waived.

\section{Study participants}

We screened all patients with the discharge diagnosis of STEMI in Surat Thani Hospital between October 2015 and September 2017, using International Statistical Classification of Diseases and Related Health Problems 10 (ICD-10) codes I21.0-I21.3. The inclusion criteria were the STEMI patients who received reperfusion therapy either primary PCI or thrombolytic agent within 12 hours of symptom onset. Patients with alternative diagnoses (including coronary vasospasm or aortic dissection) or STEMI after hospitalization were excluded.

\section{Data collection}

Patients' clinical data, coronary angiographic results, and the echocardiographic result of each admission were collected from electronic medical records. Clinical data included gender, age, smoking status, comorbidities, cardiogenic shock at presentation, Killip classification, electrocardiogram (ECG), and therapeutic profile were retrieved from each admission record.

\section{Definitions}

The diagnosis of STEMI was reviewed and confirmed by the interventional cardiologist $(\mathrm{CW})$ according to the third universal definition of MI. Cardiogenic shock at presentation was defined using the definition from SHOCK Trial including: (I) systolic blood pressure (SBP) $<90 \mathrm{mmHg}$ for $>30$ minutes or required vasopressor support to maintain SBP $>90 \mathrm{mmHg}$; (II) Evidence of end-organ damage (Urine output $<30 \mathrm{~mL} /$ hour or cool extremities); (III) Hemodynamic criteria: Cardiac index $<2.2$ and Pulmonary capillary wedge pressure $>15 \mathrm{mmHg}$. The LVEF was measured by a transthoracic echocardiogram (TTE) using the modified Simpsons or Teicholz method.

\section{Statistical analysis}

Categorical variables are presented as percentages and were 
Table 1 Comparison of clinical characteristics between non-survived and survived STEMI patients during hospitalization ( $\mathrm{n}=431$ )

\begin{tabular}{|c|c|c|c|c|}
\hline Characteristics & Overall $(n=431)$ & Survived $(n=399)$ & Dead $(n=32)$ & $P$ value \\
\hline Age (years), mean $\pm S D$ & $59.60 \pm 13.94$ & $59.23 \pm 13.95$ & $64.16 \pm 13.20$ & 0.0545 \\
\hline Diabetes, n (\%) & $100(23.20)$ & $88(22.06)$ & $12(37.50)$ & 0.0524 \\
\hline Chronic kidney disease, n (\%) & $25(5.80)$ & $20(5.01)$ & $5(15.62)$ & 0.0298 \\
\hline Previous stroke, n (\%) & $20(4.64)$ & $16(4.01)$ & $4(12.50)$ & 0.0521 \\
\hline Atrial fibrillation, n (\%) & $8(1.86)$ & $8(2.01)$ & $0(0.00)$ & 1 \\
\hline Anterior wall $\mathrm{Ml}$ at presentation, $\mathrm{n}(\%)$ & $208(48.26)$ & $186(46.62)$ & $22(68.75)$ & 0.0172 \\
\hline Cardiogenic shock at presentation, n (\%) & $129(30.07)$ & $103(25.94)$ & $26(81.25)$ & $<0.0001$ \\
\hline 1 & $268(62.47)$ & $263(66.25)$ & $5(15.62)$ & \\
\hline 2 & $17(3.96)$ & $17(4.28)$ & $0(0.00)$ & \\
\hline 3 & $15(3.50)$ & $14(3.53)$ & $1(3.12)$ & \\
\hline 4 & $129(30.07)$ & $103(25.94)$ & $26(81.25)$ & \\
\hline LVEF (\%), median [IQR] & $51.00[44.00,60.00]$ & $51.00[44.00,60.00]$ & $34.00[32.00,36.00]$ & 0.0017 \\
\hline LVEF below 40\%, n (\%) & $42(14.05)$ & 38 (12.93) & $4(80.00)$ & 0.0015 \\
\hline Total ischemic time (min), median [IQR] & $240.00[150.00,390.00]$ & $240.00[150.00,397.50]$ & $240.00[150.00,300.00]$ & 0.6611 \\
\hline PCl, n (\%) & $306(71.00)$ & $280(70.18)$ & $26(81.25)$ & 0.2267 \\
\hline
\end{tabular}

STEMI, ST-elevation myocardial infarction; SD, standard deviation; LVEF, left ventricular ejection fraction; IQR, interquartile range; IABP, intra-aortic balloon pump; $\mathrm{PCl}$, percutaneous coronary intervention.

analyzed using Fisher's exact test. Continuous variables are presented as the mean and standard deviation (SD) and using the two-sample $t$-test. Non-parametric continuous variables are presented as interquartile ranges (IQRs) and using the Wilcoxon rank-sum (Mann-Whitney) test.

All proportions and $\mathrm{P}$ values were calculated based on variables with no missing data. Logistic regression analysis was used to determine the factors associated with inhospital mortality. Odds ratios (ORs) and their $95 \%$ CIs were estimated. Variables at $\mathrm{P}<0.1$ on univariable analysis were selected for inclusion in the final multivariable logistic regression model. $\mathrm{P}<0.05$ was considered to be statistically significant in the multivariable logistic regression model.

\section{Results}

Of 500 patients with a discharge diagnosis of STEMI in Surat Thani hospital between October 2015 and September 2017, 431 patients were included into the present study. Majorities of the patients were male $(80.5 \%)$ with mean age of 59.6 years. Almost half of the population had anterior wall MI, 30.1\% had a cardiogenic shock, and $6.0 \%$ had a cardiac arrest at presentation. During the study period, 32 patients died (7.4\%), and 399 survived during the index hospitalization. The clinical characteristics of non-survived and survived patients are shown in Table 1. The prevalence of chronic kidney disease, anterior wall MI, cardiogenic shock and cardiac arrest in non-survived group was higher 
Table 2 Factors associated with in-hospital among STEMI patients on univariable and multivariable logistic regression analyses

\begin{tabular}{|c|c|c|c|c|c|c|}
\hline Factors & \multicolumn{3}{|c|}{ Univariable } & \multicolumn{3}{|c|}{ Multivariable } \\
\hline Age & 1.03 & $1.00-1.05$ & 0.0565 & 1 & $0.91-1.1$ & 0.9639 \\
\hline Diabetes mellitus & 2.12 & $1.00-4.51$ & 0.0507 & 0.5 & $0.03-8.26$ & 0.6248 \\
\hline Chronic kidney disease & 3.51 & $1.22-10.08$ & 0.0197 & 2.83 & $0.13-60.01$ & 0.5042 \\
\hline Anterior wall STEMI & 2.52 & $1.16-5.46$ & 0.0191 & 0.65 & $0.06-7.16$ & 0.723 \\
\hline Cardiac arrest & 16.5 & $6.76-40.28$ & $<0.0001$ & 22.42 & $1.22-410.53$ & 0.0361 \\
\hline Cardiogenic shock & 12.37 & $4.95-30.9$ & $<0.0001$ & 0.22 & $0.01-4.87$ & 0.3349 \\
\hline LVEF & 0.91 & $0.85-0.97$ & 0.0036 & 0.91 & $0.83-0.98$ & 0.0195 \\
\hline
\end{tabular}

STEMI, ST-elevation myocardial infarction; LVEF, left ventricular ejection fraction.

than the survived group. Echocardiography was performed in 299 patients $(69.4 \%)$ and the median LVEF in overall population was $51 \%$.

\section{Risk factors associated with in-hospital mortality}

In comparison with survived patients, non-survived patients were more likely to be chronic kidney disease $(5.01 \% \mathrm{vs}$. $15.62 \%, \mathrm{P}=0.0298)$, had anterior wall STEMI (46.62\% vs. $68.75 \%, \mathrm{P}=0.0172)$, Cardiogenic shock $(25.94 \%$ vs. $81.25 \%$, $\mathrm{P}<0.0001)$, cardiac arrest $(3.51 \%$ vs. $37.50 \%, \mathrm{P}<0.0001)$ at presentation, and had lower median LVEF (51\% vs. 34\%, $\mathrm{P}<0.0001)$ and Intra-aortic balloon pump used $(1.25 \%$ vs. $15.62 \%, \mathrm{P}=0.003)$.

In univariable analysis, the following factors were associated with in-hospital mortality (Table 2). In multivariable analysis, LVEF (aOR 0.91, 95\% CI, 0.83-0.98, $\mathrm{P}=0.0195)$ and cardiac arrest at presentation (aOR 22, 95\% CI, 1.22-410.05, $\mathrm{P}=0.0361$ ) were independently associated with in-hospital mortality.

\section{Discussion}

The prevalence of in-hospital mortality of the present study (eight percent) was less than Thai ACS registry (3) but higher when compared the study from Japanese study (8). The main reason come from the all patient received reperfusion treatment either fibrinolytic or PCI.

The previous study from china (9) found history of hyperlipidemia, T2DM, smoking and cardiogenic shock, arrhythmia, heart failure, intra-aortic balloon pump, thrombus aspiration and laboratory finding were significant higher in the death group but lack data about LVEF during hospitalization.

LVEF is well known independent specific risk factor for acute coronary syndrome and current guidelines recommend evaluate of LVEF is indicated before discharge (10).

The our study was demonstrated that the lower LVEF (equal or less than $40 \%$ ) and cardiac arrest at presentation were independent factors associated with in-hospital mortality of all comer STEMI patient. This finding was similar to the prognostic factor values for 1-year mortality in LVEF in acute coronary syndrome (both non-STEMI and STEMI) (11) and prognostic factor of in-hospital mortality in all STEMI undergoing primary PCI (12).

Therefore, echocardiogram to identify patient at a high risk of death while being hospitalized very necessary including evaluating and treating complications. The prognostic model to predict adverse outcome of STEMI patient after reperfusion should be add on from clinical presentation, severity score (13) and laboratory result (9) from previous study. The intensive treating and providing treatment after discharge should be provided especially in groups with evidence that the mortality rate could be reduced in patients with reduced left ventricular function: Angiotensin receptor antagonist, beta blocker, angiotensin receptor blocker, or mineralocorticoid receptor antagonist according to guideline (6).

In the patient was treated with ST-elevation myocardial infarction, all left ventricular function should be evaluated. In the case that any hospital was unable to performed echocardiogram while patient was admitted especially in 
developing country hospital (14), it should refer to the tertiary center which able to performed.

The results of the present study should be interpreted in the context of the following limitations. First, the study was conducted in a single tertiary center where the percentage of the patients treated with thrombolytic therapy is relatively high. Therefore, the results may not be applied to the area where the utilization rate of the primary PCI is high. However, thrombolytic therapy is still the primary reperfusion strategy in many areas of the world, in particular, the developing countries. Second, the retrospective nature of the study limits the completeness of the data collection; thereby, the missing data is unavoidable. Lastly, the assessment of LVEF was done by treating cardiologists in our hospital, and there was no assessment of the inter-observer variability between two observers. However, the data represents the information in the real-world setting, and the LVEF assessment by echocardiography was widely performed by cardiologists using standardized methods.

\section{Conclusions}

LVEF and cardiac arrest at presentation were independently associated with in-hospital mortality in STEMI patients receiving reperfusion therapy either fibrinolytic therapy or primary PCI.

\section{Acknowledgments}

Funding: The study was supported by the Suratthani Medical Education Center, Suratthani, Thailand.

\section{Footnote}

Reporting Checklist: The authors present the study in accordance with the TRIPOD reporting checklist. Available at http://dx.doi.org/10.21037/cdt-20-398

Data Sharing Statement: Available at http://dx.doi. org/10.21037/cdt-20-398

Conflicts of Interest: All authors have completed the ICMJE uniform disclosure form (available at http://dx.doi. org/10.21037/cdt-20-398). The authors have no conflicts of interest to declare.

Ethical Statement: The authors are accountable for all aspects of the work in ensuring that questions related to the accuracy or integrity of any part of the work are appropriately investigated and resolved. The study was conducted in accordance with the Declaration of Helsinki (as revised in 2013). The ethical committee of Surat Thani Hospital, Thailand, approved the study protocol (No. 19/2020) and individual consent for this retrospective analysis was waived.

Open Access Statement: This is an Open Access article distributed in accordance with the Creative Commons Attribution-NonCommercial-NoDerivs 4.0 International License (CC BY-NC-ND 4.0), which permits the noncommercial replication and distribution of the article with the strict proviso that no changes or edits are made and the original work is properly cited (including links to both the formal publication through the relevant DOI and the license). See: https://creativecommons.org/licenses/by-nc-nd/4.0/.

\section{References}

1. Thygesen K, Alpert JS, Jaffe AS, et al. Fourth universal definition of myocardial infarction (2018). Eur Heart J 2019;40:226-69.

2. Jernberg T, Held C, Rydberg E. SWEDEHEART Annual report 2014. Stockholm: Tomas Jernberg, Karolinska University Hospital, 2014.

3. Srimahachota S, Boonyaratavej S, Kanjanavanit R, et al. Thai Registry in Acute Coronary Syndrome (TRACS)an extension of Thai Acute Coronary Syndrome Registry (TACS) group: lower in-hospital but still high mortality at one-year. J Med Assoc Thai 2012;95:508-18.

4. Fox KA, Dabbous OH, Goldberg RJ, et al. Prediction of risk of death and myocardial infarction in the six months after presentation with acute coronary syndrome: prospective multinational observational study (GRACE). BMJ 2006;333:1091.

5. Morrow DA, Antman EM, Charlesworth A, et al. TIMI risk score for ST-elevation myocardial infarction: a convenient, bedside, clinical score for risk assessment at presentation: an intravenous nPA for treatment of infarcting myocardium early II trial substudy. Circulation 2000;102:2031-7.

6. Ibánez B, James S, Agewall S, et al. 2017 ESC Guidelines for the management of acute myocardial infarction in patients presenting with ST-segment elevation. Rev Esp Cardiol (Engl Ed) 2017;70:1082.

7. Nishimura RA, Reeder GS, Miller Jr FA, et al. Prognostic 
value of predischarge 2-dimensional echocardiogram after acute myocardial infarction. Am J Cardiol 1984;53:429-32.

8. Miyachi H, Takagi A, Miyauchi K, et al. Current characteristics and management of ST elevation and non-ST elevation myocardial infarction in the Tokyo metropolitan area: from the Tokyo CCU network registered cohort. Heart Vessels 2016;31:1740-51.

9. Wang R, Mei B, Liao X, et al. Determination of risk factors affecting the in-hospital prognosis of patients with acute ST segment elevation myocardial infarction after percutaneous coronary intervention. BMC Cardiovasc Disord 2017;17:243.

10. Jneid H, Addison D, Bhatt DL, et al. 2017 AHA/ACC clinical performance and quality measures for adults with ST-elevation and non-ST-elevation myocardial infarction: a report of the American College of Cardiology/American Heart Association Task Force on Performance Measures. J Am Coll Cardiol 2017;70:2048-90.

Cite this article as: Wichian C, Morasert T, Nilmoje T, Chichareon P. Prevalence and predictors associated with inhospital mortality in acute ST segment elevation myocardial infarction after reperfusion therapy in developing country. Cardiovasc Diagn Ther 2020;10(5):1264-1269. doi: 10.21037/cdt20-398
11. Brezinov OP, Klempfner R, Zekry SB, et al. Prognostic value of ejection fraction in patients admitted with acute coronary syndrome: a real world study. Medicine (Baltimore) 2017;96:e6226.

12. Kiatchoosakun S, Wongwipaporn C, Pussadhamma B. Prognostic factors of in-hospital mortality in all comers with ST elevation myocardial infarction undergoing primary percutaneous coronary intervention. Heart Asia 2016;8:13-7.

13. Hou LL, Gao C, Feng J, et al. Prognostic Factors for In-Hospital and Long-Term Survival in Patients with Acute ST-Segment Elevation Myocardial Infarction after Percutaneous Coronary Intervention. Tohoku J Exp Med 2017;242:27-35.

14. Dharma S, Andriantoro H, Dakota I, et al. Organisation of reperfusion therapy for STEMI in a developing country. Open Heart 2015;2:e000240. 